Marquette University

e-Publications@Marquette

3-6-2017

\title{
Neurocognitive Predictors of Treatment Response to Randomized Treatment in Adults with Tic Disorders
}

Amitai Abramovitch

Texas State University

Lauren S. Hallion

Massachusetts General Hospital

Hannah E. Reese

Massachusetts General Hospital

Douglas W. Woods

Marquette University, douglas.woods@marquette.edu

Alan Peterson

The University of Texas Health Science Center - San Antonio

See next page for additional authors

Follow this and additional works at: https://epublications.marquette.edu/psych_fac

Part of the Psychology Commons

\section{Recommended Citation}

Abramovitch, Amitai; Hallion, Lauren S.; Reese, Hannah E.; Woods, Douglas W.; Peterson, Alan; Walkup, John T.; Piacentini, John; Scahill, Lawrence; Deckersbach, Thilo; and Wilhelm, Sabine, "Neurocognitive Predictors of Treatment Response to Randomized Treatment in Adults with Tic Disorders" (2017).

Psychology Faculty Research and Publications. 290.

https://epublications.marquette.edu/psych_fac/290 


\section{Authors}

Amitai Abramovitch, Lauren S. Hallion, Hannah E. Reese, Douglas W. Woods, Alan Peterson, John T. Walkup, John Piacentini, Lawrence Scahill, Thilo Deckersbach, and Sabine Wilhelm 
Marquette University

e-Publications@Marquette

\section{Biology Faculty Research and Publications/College of Arts and Sciences}

This paper is NOT THE PUBLISHED VERSION; but the author's final, peer-reviewed manuscript. The published version may be accessed by following the link in the citation below.

Progress in Neuro-Pschopharmacology and Biological Psychiatry, Vol. 74, (March 6, 2017): 9-14. DOI. This article is (C) Elsevier and permission has been granted for this version to appear in ePublications@Marquette. Elsevier does not grant permission for this article to be further copied/distributed or hosted elsewhere without the express permission from Elsevier.

\section{Neurocognitive Predictors of Treatment Response to Randomized Treatment in Adults with Tic Disorders}

Amitai Abramovitch

Department of Psychology, Texas State University, San Marcos, TX

Department of Psychiatry, Massachusetts General Hospital, Boston, MA

Department of Psychiatry, Harvard Medical School, Boston, MA

Hannah E. Reese

Department of Psychiatry, Massachusetts General Hospital, Boston, MA

Department of Psychiatry, Harvard Medical School, Boston, MA

Department of Psychology, Bowdoin College, Brunswick, ME

Douglas W. Woods

Department of Psychology, Marquette University, Milwaukee, WI

Alan Peterson

The University of Texas Health Science Center at San Antonio, San Antonio, TX

John T. Walkup

Department of Psychiatry, Weill Cornell Medical College, New York, NY

John Piacentini 
Department of Psychiatry and Biobehavioral Sciences, University of California Los Angles, Los Angeles, CA

\author{
Lawrence Scahill \\ Department of Psychiatry, Yale University, New Haven, CT \\ Department of Pediatrics, Emory University, Atlanta, GA \\ Thilo Deckersbach \\ Department of Psychiatry, Massachusetts General Hospital, Boston, MA \\ Department of Psychiatry, Harvard Medical School, Boston, MA \\ Sabine Wilhelm \\ Department of Psychiatry, Massachusetts General Hospital, Boston, MA \\ Department of Psychiatry, Harvard Medical School, Boston, MA
}

\title{
Highlights
}

- The role of inhibitory functions in adult TS/CTD is unclear.

- Adolescents and adults with TS/CTD may possess intact or superior inhibitory control.

- We examined the association between baseline inhibitory function and treatment response in adults TS/CTD.

- Neuropsychological tests did not predict treatment response regardless of treatment type.

- Results support the notion that inhibitory deficits are not core facets of TS/CTD in adults.

\begin{abstract}
Tourette's disorder (TS) and chronic tic disorder (CTD) are neurodevelopmental disorders characterized by involuntary vocal and motor tics. Consequently, TS/CTD have been conceptualized as disorders of cognitive and motor inhibitory control. However, most neurocognitive studies have found comparable or superior inhibitory capacity among individuals with TS/CTD relative to healthy controls. These findings have led to the hypothesis that individuals with TS/CTD develop increased inhibitory control due to the constant need to inhibit tics. However, the role of cognitive control in TS/CTD is not yet understood, particularly in adults. To examine the role of inhibitory control in TS/CTD, the present study investigated this association by assessing the relationship between inhibitory control and treatment response in a large sample of adults with TS/CTD. As part of a large randomized trial comparing behavior therapy versus supportive psychotherapy for TS/CTD, a battery of tests, including tests of inhibitory control was administered to 122 adults with TS/CTD at baseline. We assessed the association between neuropsychological test performance and change in symptom severity, as well as compared the performance of treatment responders and non-responders as defined by the Clinical Global Impression Scale. Results indicated that change in symptoms, and treatment response were not associated with neuropsychological performance on tests of inhibitory control, intellectual ability, or motor function, regardless of type of treatment. The finding that significant change in symptom severity of TS/CTD patients is not associated with impairment or change in inhibitory control regardless of treatment type suggests that inhibitory control may not be a clinically relevant facet of these disorders in adults.
\end{abstract}

\section{Keywords}

Tourette's disorder, Response inhibition, Executive function, Treatment, Neuropsychology 


\section{Introduction}

Tourette's disorder (TS) and persistent (chronic) motor or vocal tic disorder (CTD), are neurodevelopmental disorders characterized by multiple vocal and motor tics (American Psychiatric Association, 2013). The worldwide prevalence of tic disorders in children is estimated at $0.8 \%$, with higher prevalence in boys (1.1\%), whereas the prevalence of tic disorders in adults is estimated at 1:2000 (Knight et al., 2012). Tic disorders usually onset in childhood and their severity tend to decrease with age. However, it has been estimated that $11 \%$ of individuals with tic disorders continue to experience moderate to severe tics resulting in daily life functional impairments into adulthood (Bloch et al., 2006, Leckman et al., 1998).

Compared to controls, individuals diagnosed with tic disorders exhibit different patterns of brain activity in the cortico-striato-thalamo-cortical (CSTC) neural circuitry (Leckman et al., 2010). The prominent role of the CSTC system in executive and inhibitory functions, together with the clinical presentation of tics, led to the hypothesis that tic disorders are disorders of motor disinhibition, wherein patients experience difficulties suppressing tics (Jung et al., 2013). Indeed, imaging studies reveal increased activation of the CSTC network in patients with tic disorders during attempts to inhibit eye blinking (Mazzone et al., 2010).

\subsection{Inhibitory control in tic disorders}

Studies investigating executive function in tic disorders, particularly tasks of inhibitory control (including response inhibition, response suppression, and interference control), reveal mixed results (Kalsi et al., 2015). In fact, the majority of studies utilizing the gold standard tests of inhibitory control have revealed intact performance among adults with tic disorders. These include research utilizing Go/No-Go tasks (GNG; Serrien et al., 2005, Thomalla et al., 2014, Watkins et al., 2005) and the Stroop task (Eddy and Cavanna, 2014, Thibault et al., 2009). Very few studies found underperformance on tasks of behavioral inhibition in adults with TS/CTD (Jackson et al., 2015), but these results were found on tasks such as Sentence Completion, or the Simon task (Dursun et al., 2000, Georgiou et al., 1995). In addition, it has been argued that such studies tend to include participants with comorbid disorders such as Attention Deficit/Hyperactivity Disorder (ADHD) and OCD, and that inhibitory deficits may be found mainly in individuals diagnosed with tic disorders concomitant with OCD and/or ADHD (Jung et al., 2013). Notably, recent reviews of the literature highlight findings indicating a paradoxical superior behavioral control among adolescents and adults diagnosed with tic disorders compared to controlshypothetically due to years of experience attempting to inhibit tics (Jackson et al., 2015, Jung et al., 2013). However, more research is required to support this notion, and the role of behavioral inhibition in adult TS/CTD remains unclear.

\subsection{Neuropsychological functions and treatment response in tic disorders}

Behavioral interventions for tic disorders, such as Habit Reversal Therapy (HRT) and its newer version called Comprehensive Behavioral Intervention for Tics (CBIT), are found to be effective for the treatment of tic disorders, (Piacentini et al., 2010, Wilhelm et al., 2012) yielding large effect sizes in 
adult samples (McGuire et al., 2014). However, very little is known about predictors of treatment response to behavior therapy for tic disorders. Neuropsychological predictors of treatment response may be important in informing treatment selection, as well as informing treatment development. To our knowledge, there are only three studies that examined changes in cognitive function following cognitive behavioral therapy (CBT) for adult TS/CTD. Lavoie et al. (2011) reported improved performance on the Purdue Pegboard Test following treatment. However, this test assesses basic motor functions, and the authors did not include executive function or other neuropsychological tests in their study. In another study (Deckersbach et al., 2006) the authors compared a small sample of individuals diagnosed with TS receiving Habit Reversal Therapy $(n=15)$ to a sample $(n=15)$ receiving supportive psychotherapy. The authors found that aspects of performance on a visuospatial priming task had predictive value for treatment response. Notably, the authors used this task to assess response inhibition, although the task was visuospatial in nature which poses difficulties in differentiating between the predictive value of visuospatial function versus response inhibition. In a recent study, however, Morand-Beaulieu et al. (2015) employed a stimulus-response compatibility inhibition task in a sample of 20 TS/CTD adult patients and 20 controls and found no performance difference between pre- and post-treatment. Thus, the goal of this study was to utilize gold standard tasks of inhibitory control-namely, the Go/No-Go (GNG) test assessing response inhibition, and the Stroop test, assessing interference control-to predict treatment response to CBIT among adults with TS and CTD. In light of the mixed literature and the novelty of this study, our investigation is exploratory.

\section{Materials and methods}

\subsection{Study design}

Participants were recruited as part of a large-scale, randomized controlled trial comparing 10 weeks (8 sessions) of Comprehensive Behavioral Intervention for Tics (CBIT) to psychoeducation and supportive therapy (PST). See Wilhelm et al. (2012) for a detailed description of the study procedures. Neuropsychological measures were administered at baseline. Clinical severity was assessed by an independent evaluator (a clinician blind to treatment condition) at baseline and at post-treatment.

Participants were recruited at three sites: Massachusetts General Hospital/Harvard Medical School, Yale University, and University of Texas Health Science Center at San Antonio.

\subsection{Participants}

Adult participants $(n=122)$ were included in the present study. Inclusion criteria were age $\geq 16$ years, a diagnosis of TS or CTD of moderate severity or greater based on the Clinical Global Impression-Severity Score (CGI-S $\geq 4$ ), and a Yale Global Tic Severity Scale (YGTSS; Leckman et al., 1989) total score $\geq 14$ (> 10 for those with only motor or vocal tics). Additional inclusion criteria were fluency in English, $\mathrm{IQ}>80$ on a standardized intelligence test, no history of schizophrenia or pervasive developmental disorder, and no current substance use disorder. Other comorbidities (bipolar disorder, depression, anxiety and related disorders, and ADHD) were permitted provided that the co-occurring disorder was stable and not of sufficient severity to require clinical attention. Participants were excluded if they 
previously completed a course ( $>4$ sessions) of CBT for tics. Medications for tics were permitted provided the dose was stable for at least 6 weeks with no planned changes for the duration of the study. Fifty-one participants (41.8\%) were medicated-out of which 8 participants were on tic medication only (e.g., alpha agonists, neuroleptics), 23 were on other medications in addition to tic medication, and 20 participants were on non-tic medication only (e.g., SSRIs).

\subsection{Treatments}

A comprehensive description of the study treatments can be found elsewhere (Wilhelm et al., 2012). Briefly, both treatments consisted of eight 60-90 min sessions administered over 10 weeks. CBIT comprised psychoeducation, tic awareness training, competing response training, relaxation training, and functional analysis. PST comprised disorder-specific psychoeducation and supportive therapy. Therapists had at least a master's degree in clinical psychology, followed detailed treatment manuals, and were specifically trained on both treatments for this study. Treatment sessions were videotaped and randomly selected for fidelity ratings. Fidelity was good or better for $75.7 \%$ of CBIT tapes and $87.7 \%$ of PST tapes.

\subsection{Measures}

\subsubsection{Clinical measures}

Structured Clinical Interview for DSM-IV Patient Version (SCID-P):

Diagnostic status was assessed via the SCID-P (First et al., 2002), a widely-used and well-validated semistructured interview developed to establish past and current DSM-IV diagnoses.

\section{Clinical Global Impression-Improvement Scale (CGI-I):}

The CGI-I (Guy and Bonato, 1970) is a single-item standard global assessment used to assess changes in severity of the target disorder. The CGI-I scores range between 1 (very much improved) and 7 (very much worse). Positive response to treatment in the present study was defined as a score of 2 or 1 (much improved, or very much improved). The CGI-I score in each time point was assessed by an evaluator that was blind to treatment assignment.

Yale Global Tic Severity Scale (YGTSS):

The YGTSS (Leckman et al., 1989) is a clinician-rated scale used to assess tic severity and was the primary clinical outcome measure in this study. Motor and phonic tics are rated separately from 0 to 5 on several scales including: number, frequency, intensity, complexity, and interference. Thus, motor and phonic tic scores can range from 0 to 25; the combined Total Tic score ranges from 0 to 50 . There is also an impairment score that rates the overall burden due to tics. The impairment scale yields a single score from 0 to 50 with higher scores indicating higher levels of overall impairment associated with tics. The YGTSS has demonstrated strong psychometric properties with good internal consistency, excellent inter-rater reliability, and strong convergent and divergent validity (Leckman et al., 1989). 
The primary outcome measure in this study was the Total Tic score, as it shows the greatest sensitivity to change in tic severity over brief periods of time (Lin et al., 2002).

\subsubsection{Neuropsychological tests}

\subsubsection{General tests}

Wechsler Test of Adult Reading (WTAR):

The Wechsler Test of Adult Reading (WTAR; Wechsler, 2001) provides an estimate of premorbid IQ. The WTAR shows a strong $(r>0.7)$ correlation with IQ as assessed by the Wechsler Adult Intelligence Scale (Strauss et al., 2006). Subjects are to read a list of words and receive a point for each correctly pronounced word.

Purdue Pegboard Test:

The Purdue Pegboard Test (Tiffin and Asher, 1948) measures manual dexterity. The task consists of a board with holes and a set of pegs. Subjects are instructed to place the pegs in the holes (one at a time) as quickly and correctly as possible. Outcomes can be presented separately for left, right, and both hands. For the purposes of the present paper, we report only the results for both hands. Tiffin and Asher (1948) provide a means to calculate scaled scores; however, the normative samples are comprised of adults who applied for factory and production jobs and therefore may not be representative of the general population. We therefore use raw scores in analyses and present descriptive data from a large general sample of Canadian adults (Yeudall et al., 1986) for comparison.

\subsubsection{Inhibitory control tasks}

Stroop color \& word test:

The Stroop task is a classic cognitive interference task (Stroop, 1935). It assesses inhibition of a dominant response (reading) in favor of an alternative competing response (color naming). In the first condition, participants are presented with color words (written in black) and are asked to name the color as quickly as possible. In the second condition, subjects are asked to name the color of a nonsense stimulus (XXXXX) as quickly as possible. In the Interference condition, participants are presented with color words (e.g. red) written in a color that is inconsistent with the word's meaning (e.g. red written in blue). Participants are required to name the color the word is printed in as quickly as possible. An interference (color-word) score was calculated as a standardized score based on age and education-corrected normative data.

Go/No-Go task (GNG):

The GNG task (Serrien et al., 2005) is a computerized measure of motor response inhibition. In the task, participants view a cue stimulus (an arrow pointing to the right or left) that signaled them to get ready, followed by a target stimulus (the figure 0 or the letter $S$ ) that indicated the correct response. Participants were seated in front of a desk with a custom-built button-press device held in each hand and faced a computer screen at a distance of $80 \mathrm{~cm}$. A fixation cross was visible continuously in the center of the screen. During each trial, an arrow was presented that pointed to the right or to the left 
and served as a cue for a following target. On "go" trials (75\% of trials), the target stimulus was the figure 0 . On "No-Go" (response inhibition) trials ( $25 \%$ of trials), the target signal was the letter $\mathrm{S}$, and indicated that the planned response needed to be withheld (inhibited).

The cue and target stimuli appeared on the right or left side of the fixation cross to enhance stimulusresponse compatibility and remained visible on the screen for $500 \mathrm{~ms}$; a fixed time interval of $3 \mathrm{~s}$ occurred between the onsets of cue and target. Participants responded to the target signal by pressing the right-sided or left-sided button as fast as possible with the thumb of the right or left hand (as instructed by the direction of the cue arrow and the asymmetry of the target signal with respect to the central fixation cross on screen). Commission errors were calculated as the percent of false alarms (incorrect positive responses) divided by the total number of No-Go trials ( 20 trials). Omission errors were calculated as the percent of misses (incorrect negative responses) divided by the total number of go trials (60 trials).

\subsection{Statistical analyses}

Clinical improvement was computed as the change in YGTSS total score from baseline to posttreatment (week 10). Each neuropsychological measure was examined in a separate regression model as a predictor of treatment outcome with treatment condition (CBIT vs. PST) included as a moderator. These analyses were conducted using the PROCESS macro for SPSS (Hayes, 2013). Multivariate analysis of variance was conducted in order to assess performance differences between treatment responders and non-responders on neuropsychological tasks.

\section{Results}

Participants were 122 adults ( $\mathrm{M}$ age $=31.55, \mathrm{SD}=13.72 ; 36 \%$ female) with an average total tic severity of 22.91 (SD $=6.60$ ) as assessed by the YGTSS. Participants were generally of average intelligence (WTAR FSIQ M = 106.02, SD = 9.56). Motor functioning was somewhat deficient: The average Purdue Pegboard performance for both hands was approximately one standard deviation below the mean reported in a large normative adult sample $(M=11.32, S D=2.02$ in this sample, versus norms $M=12.69, S D=1.55$; Yeudall et al., 1986), and was equivalent to scores obtained in other adult CTD and TS samples (e.g., Lavoie et al., 2007) (Table 1).

Table 1. General neuropsychological functioning as a predictor of treatment response.

WTAR FSIQ

$$
\begin{aligned}
& \text { Condition } \\
& \text { WTAR FSIQ } \\
& \text { Condition x FSIQ }
\end{aligned}
$$

Purdue Pegboard Test $\begin{array}{lllllll}\text { B } & \text { SE (B) } & t & p & 95 \% \mathrm{Cl} & R \text {-sq } & F\end{array}$

$0.186 .95^{* * *}$

$$
\begin{array}{lllll}
12.66 & 12.10 & 1.05 & 0.30-11.34-36.67 \\
-0.09 & 0.07 & -1.18 & 0.24-0.24-0.06 \\
-0.08 & 0.11 & -0.73 & 0.47-0.31-0.14
\end{array}
$$

$0.176 .54^{* * *}$
$9.66 \quad 5.72 \quad 1.68 \quad 0.09-1.69-21.01$ 


$\begin{array}{lccccr} & \text { B } & \text { SE (B) } & \boldsymbol{t} & \boldsymbol{p} & \mathbf{9 5 \%} \mathbf{~ C l} \\ \text { Both hands } & -0.17 & 0.34 & -0.50 & 0.62 & -0.85-0.51 \\ \text { Condition x both hands interaction } & -0.50 & 0.50 & -1.01 & 0.32 & -1.48-0.49\end{array}$

WTAR $=$ Wechsler Test of Adult Reading. FSIQ $=\underline{\text { Full Scale IQ. }}$ WTAR analysis $n=102$. Purdue analysis $n=103$. $* * * p<0.001$.

\subsection{Neuropsychological predictors of treatment response}

\subsubsection{General measures}

No significant association was found between neuropsychological test performance and change in symptoms (i.e., pre - post total YGTSS score) for any of the tests administered, nor did performance interact with treatment condition to predict improvement. FSIQ did not significantly predict change in symptoms $(B=-0.09, p=0.24)$, nor did FSIQ interact with treatment condition to predict change $(B=-0.08, p=0.47)$. Purdue Pegboard Task performance similarly failed to predict change in symptoms $(B=-0.17, p=0.62)$, nor did performance interact with treatment condition $(B=-0.50$, $p=0.32$ ) (Table 2). No significant differences were found on these two tasks between treatment responders and non-responders, and no significant interaction effect was found between the two treatment conditions (Table 3 ). Medication status added to the regression and MANOVA models did not alter results.

Table 2. Inhibition as a predictor of treatment outcome.

Stroop task

$$
\begin{array}{lllllll}
\text { B } & S E(B) & t & p & 95 \% \mathrm{Cl} & R^{2} & F
\end{array}
$$

Stroop word score

Condition
Word t-score
Condition $\mathrm{x}$ word t-score

$\begin{array}{lllll}2.53 & 3.90 & 0.65 & 0.52 & -5.21-10.28 \\ -0.06 & 0.06 & -1.03 & 0.31-0.18-0.06 \\ 0.03 & 0.08 & 0.33 & 0.74 & -0.13-0.19\end{array}$

Stroop color score

Condition
Color t-score
Condition x color t-score

$4.09 \quad 4.36 \quad 0.94 \quad 0.35-4.55-12.74$

$\begin{array}{lllll}-0.07 & 0.07 & -1.06 & 0.29 & -0.21-0.06\end{array}$

$\begin{array}{lllll}-0.01 & 0.09 & -0.07 & 0.95 & -0.19-0.18\end{array}$

Stroop interference

Condition
Interference t-score
Condition $\mathrm{x}$ interference

$8.88 \quad 6.47 \quad 1.37 \quad 0.17-3.95-21.72$

$\begin{array}{lllll}-0.02 & 0.09 & -0.18 & 0.86 & -0.19-0.16\end{array}$

$\begin{array}{lllll}-0.10 & 0.12 & -0.78 & 0.44 & -0.34-0.15\end{array}$

Go/NoGo task

Commission errors

$\begin{array}{llllll}\text { Condition } & 4.20 & 1.29 & 3.27^{* * *} & 0.00 & 1.65-6.75 \\ \text { Commission errors } & 0.01 & 0.04 & 0.19 & 0.85 & -0.07-0.09 \\ \text { Condition x commission errors } & -0.02 & 0.06 & -0.30 & 0.76 & -0.14-0.11\end{array}$




\section{Omission errors}

$\begin{array}{llllll}\text { Condition } & 4.08 & 1.10 & 3.71^{* * *} & 0.00 & 1.90-6.26 \\ \text { Omission errors } & 0.02 & 0.05 & 0.45 & 0.66 & -0.08-0.12 \\ \text { Condition x omission errors } & -0.03 & 0.10 & -0.26 & 0.79 & -0.21-0.16\end{array}$

$0.145 .11^{* * *}$

Stroop analyses $n=104$. Go/NoGo analyses $n=100$.

$* * * p<0.001$.

Table 3. Neuropsychological test performance as a function of treatment response and treatment type.

\begin{tabular}{ccccccccc} 
& \multicolumn{2}{c}{ Responders } & \multicolumn{2}{c}{ Non-responders } & \multicolumn{2}{c}{ Group comparison } & \multicolumn{2}{c}{ Interaction Tx type } \\
& $\mathbf{M}$ & SD & $\mathbf{M}$ & SD & $\mathbf{F}$ & $\boldsymbol{\eta}^{2}$ & & $\mathbf{F}$ \\
WTAR FSIQ & 106.04 & 8.85 & 107.5 & 9.13 & 0.44 & 0.01 & 0.12 \\
Purdue Pegboard & 11.00 & 2.08 & 11.61 & 11.61 & 3.89 & 0.05 & 0.75 \\
Stroop word T score & 44.69 & 12.99 & 47.40 & 12.66 & 0.41 & 0.01 & 0.42 \\
Stroop color T score & 43.81 & 11.53 & 46.74 & 10.89 & 1.30 & 0.02 & 0.11 \\
Stroop interference T score & 52.12 & 5.69 & 52.37 & 9.39 & 0.37 & 0.01 & 0.45 \\
Go/NoGo commissions (\#) & 2.50 & 2.73 & 2.43 & 3.45 & 0.58 & 0.02 & 1.74 \\
Go/NoGo omissions (\#) & 1.08 & 1.60 & 2.44 & 5.31 & 1.75 & 0.05 & 0.19
\end{tabular}

WTAR $=$ Wechsler Adult Test for Reading. $\underline{F S I Q}=$ Full Scale IQ $. \eta^{2}=$ unadjusted Eta squared. Interaction Tx type = difference between responders and non-responders as a function of treatment type. \# = raw number of hits. Comparison sample sizes: WTAR - 75, 27; Purdue - 76, 27; Stroop - 77, 27; Go/NoGo - 73, 26.

\subsubsection{Inhibitory control tasks}

Stroop interference did not predict change in symptoms $(B=0.02, p=0.86)$ or interact with treatment condition to predict change $(B=0.10, p=0.44)$. Similarly, $G N G$ commission errors did not predict change in YGTSS $(B=-0.01, p=0.85)$ or interact with treatment condition to predict change $(B=0.02$, $p=0.76)$. GNG omission errors did not predict change in YGTSS $(B=-0.02, p=0.66)$ or interact with treatment condition to predict change $(B=0.03, p=0.79)$ (Table 2). Finally, no significant differences were found on inhibitory tasks between treatment responders and non-responders, and no significant interaction effect was found between the two treatment conditions (Table 3 ). Medication status added to the regression and MANOVA models did not alter results.

\section{Discussion}

To our knowledge, this is the first large scale study to investigate the predictive validity of neuropsychological tests of response inhibition on treatment response for tic disorders in adults. Our results show no significant association between performance on the Stroop or GNG tests and treatment response, regardless of the type of treatment (i.e., CBIT and PST). Moreover, no association was found between general intellectual ability or psychomotor functioning and treatment response. These null findings were evident when assessed using change in symptoms severity as a continuous dependent variable, as well as when assessed using the binary CGI-I criterion. Two previous studies 
examined the association between neuropsychological test performance and treatment response in TS/CTD samples. In contrast to our findings, Lavoie et al. (2011) reported improved performance on the Purdue Pegboard Test following CBT in a sample of TS patients. However, the sample size in their study was very small $(n=10)$. Similarly, Deckersbach et al. (2006) found some predictive validity of a visuospatial priming task for treatment response in participants with TS. However, the study had a small sample, and it utilized a visuospatial response inhibition task that is heavily influenced by visuospatial abilities that may mask inhibitory function. In contrast, our results are in accord with a more recent study that reported no association between treatment repsonse and performance on a stimulus-response compatibility inhibition task in a sample of 20 TS/CTD patients (Morand-Beaulieu et al., 2015).

The unique clinical presentation of patients with tic disorders could be perceived as a control deficit, and one might assume that these individuals suffer from substantial cognitive deficits, particularly in executive function such as inhibitory control. The results of the relatively small body of research are inconsistent, however. In fact, the majority of studies utilizing well-validated measures of executive function in TS/CTD samples indicated intact performance compared to controls (e.g., Eddy and Cavanna, 2014, Serrien et al., 2005, Thibault et al., 2009, Thomalla et al., 2014). Indeed, recent reviews of inhibitory control in TS conclude that there is no convincing evidence of deficits in inhibitory control (or executive functions) in TS/CTD (Jackson et al., 2015, Jung et al., 2013). In light of studies exhibiting enhanced motor inhibition in TS/CTD, it has been suggested that "This finding is consistent with the proposal that the frequent need to actively suppress tics leads to a generalised enhancement in the efficacy of volitional control mechanisms in TS that extends to laboratory tasks of cognitive control of motor output." (Jung et al., 2013, p. 1017).

It should be noted, however, that it has been suggested that prevalent comorbid conditions such as $\underline{\text { ADHD }}$ act as protective factors against meaningful cognitive deficits in individuals diagnosed with TS (for a review see Kalsi et al., 2015). However, this notion - that has been based on a single study assessing pediatric samples (Sukhodolsky et al., 2010), is theoretically problematic given the strong association between ADHD and cognitive deficits, and due to the need for more research directly examining this hypothesis, particularly in adults. Although more research is needed, if executive functions are indeed intact among individuals diagnosed with TS/CTD, while tic severity can be substantial and even impairing, it would be reasonable to assume that these cognitive functions would be ineffective predictors of treatment response. In fact, our results of lack of an association between change in the core symptoms of TS/CTD and baseline response inhibition lends support to the notion that tics are not a direct result of failure of motor nor cognitive inhibition. It is important to note, however, that research into neuropsychological performance as predictors of treatment response in other disorders where neuropsychological deficits are more pronounced is inconsistent, and overall neurocognitive functions do not appear to be a promising domain in terms of predictions of treatment response (e.g., Braga et al., 2016, Douglas et al., 2011, Granholm et al., 2008, Moritz et al., 2005, Wild and Gur, 2008).

The current study has several strengths. First, to our knowledge, this study is the largest to date to provide neuropsychological data on adults with TS/CTD. Second, the present sample underwent a rigorous screening procedure, and was provided with a disorder-specific evidence based treatment. 
Third, examination of the association between neuropsychological test performance and treatment response, may provide more meaningful insight compared to a cross-sectional study. Nevertheless, the present study is not without limitations. First, the present study did not include a non-clinical control sample, which hinders direct conclusions concerning an objective deficit on neuropsychological tasks. However, the WTAR and the Stroop tests produced scaled scores indicating performance on the normative range, and participants' performance on the Purdue Pegboard was found to be one standard deviation below the norms and similar to performance scores reported in other TS/CTD studies. In addition, the present study focused on specific executive function indices tapping response inhibition (GNG) and interference control (Stroop), and no other executive function, for which results may be theoretically different.

\section{Conclusion}

In the largest sample to date to assess neuropsychological predictors of treatment response in adult individuals diagnosed with TS/CTD, we found no association between neurocognitive functioning and treatment response, regardless of treatment type. These results were similar when treatment response was assessed as change in symptom severity, and when assessed using the definition of treatment response according the CGI-I. In addition, scaled scores derived from test means indirectly indicated that participants largely performed in the normative range. These results support recent research suggesting that inhibitory control deficits do not play a central role in TS/CTDs psychopathology. However, neuropsychological investigations in adult TS/CTD are limited, and more research is needed in order to provide clearer insight into neurocognitive functioning in these disorders.

\section{Ethical statement}

The present study met the highest ethical standards according to the Declaration of Helsinki, and NIMH guidelines, including obtaining informed consent from all participants, and adhering to the privacy rights of participants.

\section{Acknowledgment}

This work was supported by grants from the National Institute of Mental Health (NIMH) to Drs.

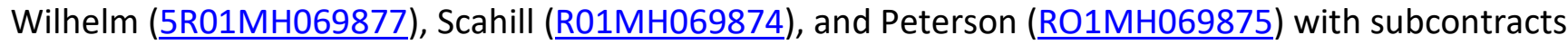
to Drs. Piacentini and Woods.

\section{References}

1 American Psychiatric Association Diagnostic and Statistical Manual of Mental Disorders: DSM-5 (5th ed.) American Psychiatric Association, Arlington, Va. (2013)

2 M.H. Bloch, B.S. Peterson, L. Scahill, J. Otka, L. Katsovich, H. Zhang, J.F. Leckman Adulthood outcome of tic and obsessive-compulsive symptom severity in children with Tourette syndrome Arch. Pediatr. Adolesc. Med., 160 (1) (2006), pp. 65-69, 10.1001/archpedi.160.1.65 
3 D.T. Braga, A. Abramovitch, L.F. Fontenelle, Y.A. Ferrao, J.B. Gomes, A.S. Vivan, ..., A.V. Cordioli Neuropsychological predictors of treatment response to cognitive behavioral group therapy in obsessive-compulsive disorder Depress. Anxiety (2016), 10.1002/da.22509

4 T. Deckersbach, S. Rauch, U. Buhlmann, S. Wilhelm Habit reversal versus supportive psychotherapy in Tourette's disorder: a randomized controlled trial and predictors of treatment response Behav. Res. Ther., 44 (8) (2006), pp. 1079-1090, 10.1016/j.brat.2005.08.007

5 K.M. Douglas, R.J. Porter, R.G. Knight, P. Maruff Neuropsychological changes and treatment response in severe depression Br. J. Psychiatry, 198 (2) (2011), pp. 115-122, 10.1192/bjp.bp.110.080713

6 S.M. Dursun, J.G. Burke, M.A. Reveley Antisaccade eye movement abnormalities in Tourette syndrome: evidence for cortico-striatal network dysfunction? J. Psychopharmacol., 14 (1) (2000), pp. 37-39

7 C.M. Eddy, A.E. Cavanna Set-shifting deficits: a possible neurocognitive endophenotype for Tourette Syndrome without ADHD J. Atten. Disord. (2014), 10.1177/1087054714545536

8 M.B. First, R.L. Spitzer, M. Gibbon, J.B.W. Williams Structured Clinical Interview for DSM-IV-TR Axis I Disorders, Research Version (Patient ed.), Biometrics Research, New York (2002)

9 N. Georgiou, J.L. Bradshaw, J.G. Phillips, J.A. Bradshaw, E. Chiu The Simon effect and attention deficits in Gilles de la Tourette's syndrome and Huntington's disease Brain, 118 (Pt 5) (1995), pp. $1305-1318$

10 E. Granholm, J.R. McQuaid, P.C. Link, S. Fish, T. Patterson, D.V. Jeste Neuropsychological predictors of functional outcome in Cognitive Behavioral Social Skills Training for older people with schizophrenia Schizophr. Res., 100 (1-3) (2008), pp. 133-143, 10.1016/j.schres.2007.11.032

11 W. Guy, R.R. Bonato CGI: Clinical Global Impression National Institute of Mental Health, Chavy Chase, MD (1970)

12 A.F. Hayes Introduction to Mediation, Moderation, and Conditional Process Analysis: A RegressionBased Approach Guilford Press (2013)

13 G.M. Jackson, A. Draper, K. Dyke, S.E. Pépés, S.R. Jackson Inhibition, disinhibition, and the control of action in Tourette Syndrome Trends Cogn. Sci., 19 (11) (2015), pp. 655-665, 10.1016/i.tics.2015.08.006

14 J. Jung, S.R. Jackson, A. Parkinson, G.M. Jackson Cognitive control over motor output in Tourette syndrome Neurosci. Biobehav. Rev., 37 (6) (2013), pp. 1016-1025, 10.1016/i.neubiorev.2012.08.009

15 N. Kalsi, R. Tambelli, P. Aceto, C. Lai Are motor skills and motor inhibitions impaired in Tourette syndrome? A review J. Exp. Neurosci., 9 (2015), pp. 57-65, 10.4137/JEN.S25095

16 T. Knight, T. Steeves, L. Day, M. Lowerison, N. Jette, T. Pringsheim Prevalence of tic disorders: a systematic review and meta-analysis Pediatr. Neurol., 47 (2) (2012), pp. 77-90, 10.1016/i.pediatrneurol.2012.05.002

17 M.E. Lavoie, T.V. Imbriglio, E. Stip, K.P. O'Connor Neurocognitive changes following cognitivebehavioral treatment in Tourette syndrome and chronic tic disorder Int. J. Cogn. Ther., 4 (1) (2011), pp. 34-50, 10.1521/ijct.2011.4.1.34 
18 M.E. Lavoie, G. Thibault, E. Stip, K.P. O'Connor Memory and executive functions in adults with Gilles de la Tourette syndrome and chronic tic disorder Cogn. Neuropsychiatry, 12 (2) (2007), pp. $165-181, \underline{10.1080 / 13546800600826371}$

19 J.F. Leckman, M.H. Bloch, M.E. Smith, D. Larabi, M. Hampson Neurobiological substrates of Tourette's disorder J. Child Adolesc. Psychopharmacol., 20 (4) (2010), pp. 237-247, 10.1089/cap.2009.0118

20 J.F. Leckman, M.A. Riddle, M.T. Hardin, S.I. Ort, K.L. Swartz, J. Stevenson, D.J. Cohen The Yale Global Tic Severity Scale: initial testing of a clinician-rated scale of tic severity J. Am. Acad. Child Adolesc. Psychiatry, 28 (4) (1989), pp. 566-573, 10.1097/00004583-198907000-00015

21 J.F. Leckman, H. Zhang, A. Vitale, F. Lahnin, K. Lynch, C. Bondi, ..., B.S. Peterson Course of tic severity in Tourette syndrome: the first two decades Pediatrics, 102 (1) (1998), pp. 14-19

22 H. Lin, C.B. Yeh, B.S. Peterson, L. Scahill, H. Grantz, D.B. Findley, ..., J.F. Leckman Assessment of symptom exacerbations in a longitudinal study of children with Tourette's syndrome or obsessive-compulsive disorder J. Am. Acad. Child Adolesc. Psychiatry, 41 (9) (2002), pp. 10701077, 10.1097/00004583-200209000-00007

23 L. Mazzone, S. Yu, C. Blair, B.C. Gunter, Z. Wang, R. Marsh, B.S. Peterson An FMRI study of frontostriatal circuits during the inhibition of eye blinking in persons with Tourette syndrome Am. J. Psychiatry, 167 (3) (2010), pp. 341-349, 10.1176/appi.ajp.2009.08121831

24 J.F. McGuire, J. Piacentini, E.A. Brennan, A.B. Lewin, T.K. Murphy, B.J. Small, E.A. Storch A metaanalysis of behavior therapy for Tourette Syndrome J. Psychiatr. Res., 50 (2014), pp. 106-112, 10.1016/i.jpsychires.2013.12.009

25 S. Morand-Beaulieu, K.P. O'Connor, G. Sauve, P.J. Blanchet, M.E. Lavoie Cognitive-behavioral therapy induces sensorimotor and specific electrocortical changes in chronic tic and Tourette's disorder Neuropsychologia, 79(Pt B) (2015), pp. 310-321, 10.1016/j.neuropsychologia.2015.05.024

26 S. Moritz, M. Kloss, D. Jacobsen, S. Fricke, C. Cutler, S. Brassen, I. Hand Neurocognitive impairment does not predict treatment outcome in obsessive-compulsive disorder Behav. Res. Ther., 43 (6) (2005), pp. 811-819

27 J. Piacentini, D.W. Woods, L. Scahill, S. Wilhelm, A.L. Peterson, S. Chang, ..., J.T. Walkup Behavior therapy for children with Tourette disorder: a randomized controlled trial JAMA, 303 (19) (2010), pp. 1929-1937, 10.1001/jama.2010.607

28 D.J. Serrien, M. Orth, A.H. Evans, A.J. Lees, P. Brown Motor inhibition in patients with Gilles de la Tourette syndrome: functional activation patterns as revealed by EEG coherence Brain, 128 (Pt 1) (2005), pp. 116-125, 10.1093/brain/awh318

29 S. Strauss, E.M. Sherman, O. Spreen A Compendium of Neuropsychological Tests: Administration, Norms, and Commentary Oxford University Press, New York (2006)

30 J.R. Stroop Studies of interference in serial verbal reactions J. Exp. Psychol., 18 (1935), pp. 643-662

31 D.G. Sukhodolsky, A. Landeros-Weisenberger, L. Scahill, J.F. Leckman, R.T. Schultz Neuropsychological functioning in children with Tourette syndrome with and without attention-deficit/hyperactivity disorder J. Am. Acad. Child Adolesc. Psychiatry, 49 (11) (2010), pp. 1155-1164, 10.1016/i.jaac.2010.08.008 
32 G. Thibault, K.P. O'Connor, E. Stip, M.E. Lavoie Electrophysiological manifestations of stimulus evaluation, response inhibition and motor processing in Tourette syndrome patients Psychiatry Res., 167 (3) (2009), pp. 202-220, 10.1016/i.psychres.2008.03.021

33 G. Thomalla, M. Jonas, T. Baumer, H.R. Siebner, K. Biermann-Ruben, C. Ganos, ..., A. Munchau Costs of control: decreased motor cortex engagement during a Go/NoGo task in Tourette's syndrome Brain, 137 (Pt 1) (2014), pp. 122-136, 10.1093/brain/awt288

34 J. Tiffin, E.J. Asher The Purdue pegboard; norms and studies of reliability and validity J. Appl. Psychol., 32 (3) (1948), pp. 234-247

35 L.H. Watkins, B.J. Sahakian, M.M. Robertson, D.M. Veale, R.D. Rogers, K.M. Pickard, ..., T.W. Robbins Executive function in Tourette's syndrome and obsessive-compulsive disorder Psychol. Med., 35 (4) (2005), pp. 571-582

36 D. Wechsler WTAR: Wechsler Test of Adult Reading Manual Psychological Corporation, San Antonio, TX (2001)

$37 \mathrm{~J}$. Wild, R.C. Gur Verbal memory and treatment response in post-traumatic stress disorder Br. J. Psychiatry, 193 (3) (2008), pp. 254-255, 10.1192/bjp.bp.107.045922

38 S. Wilhelm, A.L. Peterson, J. Piacentini, D.W. Woods, T. Deckersbach, D.G. Sukhodolsky, ..., L. Scahill Randomized trial of behavior therapy for adults with Tourette syndrome Arch. Gen. Psychiatry, 69 (8) (2012), pp. 795-803, 10.1001/archgenpsychiatry.2011.1528

39 L.T. Yeudall, D. Fromm, J.R. Reddon, W.O. Stefanyk Normative data stratified by age and sex for 12 neuropsychological tests J. Clin. Psychol., 42 (6) (1986), pp. 918-946, 10.1002/10974679(198611)42:6<918::AID-JCLP2270420617>3.0.CO;2-Y 